\title{
Visceral adiposity index is closely associated with albuminuria in the Chinese population with prediabetes: results from the REACTION study
}

Jie Wang

Chinese PLA General Hospital https://orcid.org/0000-0003-4706-0302

Binruo Zhu

Chinese PLA General Hospital

Xinye Jin

Chinese PLA General Hospital

Kang Chen

Chinese PLA General Hospital

Wenhua Yan

Chinese PLA General Hospital

Anping Wang

Chinese PLA General Hospital

\section{Weiqing Wang}

Shanghai Jiao Tong University Medical School Affiliated Ruijin Hospital

\section{Zhengnan Gao}

Dalian Municipal Central Hospital Affiliated of Dalian Medical University

Xulei Tang

Lanzhou University First Affiliated Hospital

\section{Li Yan}

Sun Yat-sen Memorial Hospital, Sun Yat-sen University

\section{Qin Wan}

The Affiliated Hospital of Southwest Medical University

\section{Zuojie Luo}

The First Affiliated Hospital of Guangxi Medical University

\section{Guijun Qin}

The First Affiliated Hospital of Zhengzhou University

\section{Lulu Chen}

Tongji Hospital of Tongji Medical College of Huazhong University of Science and Technology

Yiming Mu ( $\triangle$ muyiming@301hospital.com.cn )

https://orcid.org/0000-0002-3344-3540 
Original investigation

Keywords: visceral adiposity, albuminuria, prediabetes, Chinses population

Posted Date: May 20th, 2020

DOI: https://doi.org/10.21203/rs.3.rs-28851/v1

License: (c) (i) This work is licensed under a Creative Commons Attribution 4.0 International License. Read Full License 


\section{Abstract}

Background Visceral obesity is a major health issue and a risk factor for atherogenic state. It has been reported to be a crucial link between albuminuria and cardiovascular diseases (CVD). However, there is limited available data on the relationship between visceral obesity and albuminuria. Therefore, we aimed to explore the association between visceral obesity and albuminuria in the Chinses population with prediabetes.

Methods This cross-sectional study included 24871 prediabetic participants aged over 40 years from seven centers across China. Visceral adiposity index (VAl), which has been confirmed as a simple and reliable indicator of visceral adiposity distribution and dysfunction, is determined based on the measurements of anthropometric indices and lipid parameters. Increased albuminuria was defined as urinary albumin-creatinine ratio (UACR) $\geq 30 \mathrm{mg} / \mathrm{g}$, indicating kidney damage. Propensity score matching was used to reduce the bias and multiple logistic regression model was performed to evaluate the association between visceral obesity and albuminuria in the population with prediabetes.

Results Participants with increased UACR had increased VAl, age, blood pressure, triglycerides, poor glycemic control, and decreased estimated glomerular filtration rate (eGFR). The prevalence of CVD was higher in the increased UACR group. Multiple logistic regression analysis showed that VAl quartiles was positively associated with increased risk of albuminuria (Q2: odds rate (OR):1.10, 95\% confidence intervals [Cl]: 0.96-1.25; Q3: OR:1.16, 95\% Cl 1.01-1.32; Q4: OR:1.26, 95\% Cl 1.10-1.44, P for trend=0.001). Stratified analysis revealed that the association of VAI level with increased albuminuria risk was also observed in people who were young, women, overweight or obese, with poor control of blood pressure, and $\mathrm{eGFR} \geq 90 \mathrm{~mL} / \mathrm{min}$ per $1.73 \mathrm{~m} 2$.

Conclusions Visceral obesity assessed by VAI was significantly associated with increased albuminuria in the Chinese population with prediabetes.

\section{Background}

Urinary albumin-creatinine ratio (UACR) is not only a significant indicator of renal damage, but also has been shown to be associated with adverse renal and cardiovascular outcomes ${ }^{[1,2]}$. Data from population studies showed that UACR is an accurate predictor of kidney and cardiovascular events ${ }^{[3,4]}$. In addition, UACR is not only regarded as an initial manifestation of diabetic nephropathy, but also an early marker of atherogenic milieu in patients with type 2 diabetes mellitus (T2DM) ${ }^{[5]}$. Several studies confirmed that increased UACR is closely associated with the risk of cardiovascular-related mortality both in non-diabetic or diabetic subjects, even in the general population ${ }^{[1,6]}$. The HOPE study of 9043 participants demonstrated that increased UACR even within normal range is a risk factor for cardiovascular diseases (CVD) events in subjects with or without T2DM and the increased risk is a continuum ${ }^{[6]}$. Therefore, it is significant and necessary to detect UACR and identify individuals at high risk for routine screening in clinical practice. 
Although the underlying mechanisms between UACR and CVD events are multifactorial and have not been fully understood, obesity is thought to serve as an important intermediate link. It is established that obesity plays an important role in the onset and development of chronic kidney diseases (CKD), owing to a common core pathophysiological mechanism ${ }^{[7]}$. Compared with general obesity, visceral obesity has a higher predictive value ${ }^{[8]}$. It has been reported that visceral obesity is closely related with atherosclerosis and cardiometabolic risks ${ }^{[9,10]}$. Visceral obesity can increase the adipocytokine production, resulting in glomerular sclerosis and renal dysfunction ${ }^{[11]}$. A novel visceral adiposity index (VAl) was proposed based on body mass index (BMI), waist circumference (WC), triglycerides (TG) and high-density lipoprotein $(\mathrm{HDL})$ to estimate the distribution of visceral fat and adiposity dysfunction ${ }^{[8]}$. A robust body of literatures have confirmed the predictive and discriminative ability of VAI for unfavorable outcomes in CVD events ${ }^{[8,}$ ${ }^{12-14]}$. Recently, a cross-sectional study of 9916 subjects was aimed to explore the association between VAl and increased urinary albumin excretion. However, this study did not exclude the participants with related kidney diseases or using related drugs at baseline, possibly resulting in skew results ${ }^{[15]}$.

Visceral obesity is a key factor between albuminuria and CVD events in population at high risk for diabetes. However, few studies are available on the relationship between VAl and albuminuria in population with prediabetes. Thus, we aimed to explore the association between VAI and albuminuria in the population with prediabetes.

\section{Methods}

\section{participants and study design}

This cross-sectional study was drawn from the REACTION study, which was conducted to demonstrate the association of T2DM and prediabetes with the risk of cancer in the Chinese population. Details of the REACTION study have been described previously ${ }^{[16]}$. This cross-sectional study used the baseline investigation data from seven centers across China. A total number of 47808 participants aged over 40 years were recruited in the present study from May 2011 to December 2011. Participants, diagnosed with T2DM, kidney or hepatic diseases, cancer, using lipid-lowing drugs, antihypertensive drugs, hypoglycemic drugs, ACEI and ARB drugs and with missing data were excluded. Finally, 28071 participants with prediabetes were enrolled. Given the difference in the baseline characteristics in two groups, propensity matching was used to reduce potential bias. Finally, 24871 eligible participants were included in the final analysis (Fig. 1).

Before the study, the staff received the uniform training including the standardized questionnaire and anthropometric measurements. The study was conducted according to Declaration of Helsinki, and the protocol was approved by the Clinical Research Ethics Committee of Rui-Jin Hospital affiliated with the School of Medicine, Shanghai Jiao Tong University. Informed consents were provided by all participants before the study.

\section{Data collection and measurements}


Information were collected by using a standardized questionnaire, including medical history, smoking habits, drinking habits, previous and current use of medicine, demographics and family history. Smoking habits were classified as never, occasional (who smoked less than one cigarette per day or less than 7 cigarettes per week) and regular smokers (who smoked at least one cigarette per day). Drinking habits were classified as never, occasional (who drank less than once a week) and regular drinkers (who drank at least once a week for over six months).

Anthropometric measurements were performed by the same well-trained staff. All participants were required to wear light clothes and take off shoes when body weight and height were measured. WC were measured between the inferior costal margin and the superior border of iliac crest when participants were at the end of expiration. Body height, WC and weight were recorded to the nearest $0.1 \mathrm{~cm}$ and $0.1 \mathrm{~kg}$. BMI was calculated based on the following formula: BMI = body weight $/$ height ${ }^{2}\left(\mathrm{~kg} / \mathrm{m}^{2}\right)$. Blood pressure was measured three times by the same well-trained staff at 5-minute intervals when participants were in a seated position for at least 5-minutes rest. The mean of blood pressure was calculated and used in the final analysis.

Venous blood samples were collected in the morning after at least 10 hours overnight fasting. All participants underwent a $75 \mathrm{~g}$ oral glucose tolerance test. After 2 hours, the second venous blood samples were obtained by the same well-trained nurses. Fasting plasma glucose (FPG), $2 \mathrm{~h}$ post-load blood glucose (PBG), glycosylated hemoglobin (HbA1c), TG, total cholesterol (TC), HDL, low density lipoprotein cholesterol (LDL), gamma-glutamyl transferase (GGT), aspartate aminotransferase (AST), alanine aminotransferase (ALT), creatinine ( $\mathrm{Cr}$ ) were measured in every center, respectively.

On the basis of the Chronic Kidney Disease Epidemiology Collaboration (CKD-EPI), estimated glomerular filtration rate (eGFR) was calculated ${ }^{[17]}$. According to the criteria published by $\mathrm{WHO}$, prediabetes was divided into three subgroups as follows: (1) impaired fasting glucose (IFG): $6.1 \leq \mathrm{FBG}<7.0 \mathrm{mmol} / \mathrm{L}$ and PBG < $7.8 \mathrm{mmol} / \mathrm{L}$; (2) impaired glucose tolerance (IGT): $\mathrm{FBG}<6.1 \mathrm{mmol} / \mathrm{L}$ and $7.8 \leq \mathrm{PBG}<11.1 \mathrm{mmol} / \mathrm{L}$; and (3) IFG + IGT: $6.1 \leq \mathrm{FBG}<7.0 \mathrm{mmol} / \mathrm{L}$ and $7.8 \leq \mathrm{PBG}<11.1 \mathrm{mmol} / \mathrm{L}$.

\section{Definition of VAl and increased albuminuria}

The VAI was determined by gender-specific equations and calculated using the following formulas, where WC is expressed in $\mathrm{cm}$ and TG and $\mathrm{HDL}$ are expressed in $\mathrm{mmol} / \mathrm{L}$. Males: $[\mathrm{WC} /(39.68+(1.88 * \mathrm{BMI}))]^{*}$ $(\mathrm{TG} / 1.03) *(1.31 / \mathrm{HDL}) ;$ Females:[WC/(36.58+(1.89*BMI) $)]^{\star}(\mathrm{TG} / 0.81) *(1.52 / \mathrm{HDL})$. The first morning urine specimens were collected for urinalysis. The urinary albumin concentration and creatinine were determined by chemiluminescence immunoassay, and UACR was calculated by the following formula: urinary albumin $(\mathrm{mg})$ /urinary creatinine $(\mathrm{g})$. The seven centers used the same range and units of measurement. Based on the KDIGO CKD guideline, increased albuminuria was defined as UACR $\geq$ $30 \mathrm{mg} / \mathrm{g}$, indicating kidney damage ${ }^{[18]}$. UACR was divided into two groups as follows, normoalbuminuria: $<30 \mathrm{mg} / \mathrm{g}$ and increased albuminuria: UACR $\geq 30 \mathrm{mg} / \mathrm{g}$. VAl was divided in quartiles for the analysis. 


\section{Statistical analysis}

The statistical analysis was performed using Empower(R) (www.empowerstats.com, X\&Y Solutions Inc., Boston, MA) and R (http://www.Rproject.org). Given the difference in the baseline characteristics between eligible participants in two groups of UACR, propensity score matching was used to control potential bias. Matching was performed with the use of 1:7 matching protocol to match all covariates, with a caliper width equal to 0.05 of the standard deviation of the logit of the propensity score.

Continuous variables with a non-normal distribution were presented as median (25th percentile-75th percentile), those with a normal distribution expressed as mean \pm standard deviations. Categorical variables were presented as percentage (\%). Differences in the continuous variables between the two groups of UACR were compared using the Kruskal-Wallis test, and categorical variables were analyzed by the chi-square test. Logistic regression analysis with unadjusted and multivariate-adjusted models were conducted to determine the association between VAI quartiles and the risk of increased albuminuria. Variables considered as conventional risk factors were adjusted. Confounding factors that will change the matched odds ratio (OR) by at least $10 \%$ if added to the model were also selected for adjustment. Model 1 was unadjusted. Model 2 was adjusted for age, sex and centers. Model 3 was additionally adjusted for BMI, ALT, AST, eGFR based on Model 2. Model 4 was further adjusted for smoking, drinking, CVD events based on Model 3. Model 5 was additionally adjusted for SBP, DBP, LDL, HbA1c based on Model 4 . OR and the corresponding $95 \%$ confidence intervals $(95 \% \mathrm{Cl})$ were calculated.

To further test the association between VAI quartiles and the risk of increased albuminuria, stratified analyses were performed with feasible factors including gender (women and men), age ( $₫ 60$ and $\geq$ 60 years), weight (Underweight: BMI $<18.5 \mathrm{~kg} / \mathrm{m}^{2}$, Normal:18.5 $\leq \mathrm{BMl}<24 \mathrm{~kg} / \mathrm{m}^{2}$, Overweight: $24 \leq \mathrm{BMl}<$ $28 \mathrm{~kg} / \mathrm{m}^{2}$, Obesity: BMI $\geq 28 \mathrm{~kg} / \mathrm{m}^{2}$ ), blood pressure (Normal: systolic blood pressure $(\mathrm{SBP})<120$ and diastolic blood pressure (DBP) $<80 \mathrm{mmHg}$, High-normal: $120 \leq \mathrm{SBP}<140$ and/ or $80 \leq \mathrm{DBP}<90 \mathrm{mmHg}$, Hypertension: SBP $\geq 140$ or DBP $\geq 90 \mathrm{mmHg}$ ), prediabetes (IFG: $6.1 \leq \mathrm{FBG}<7.0 \mathrm{mmol} / \mathrm{L}$ and $\mathrm{PBG}<$ $7.8 \mathrm{mmol} / \mathrm{L}, \mathrm{IGT}: \mathrm{FBG}<6.1 \mathrm{mmol} / \mathrm{L}$ and $7.8 \leq \mathrm{PBG}<11.1 \mathrm{mmol} / \mathrm{L}, \mathrm{IFG}+\mathrm{IGT}: 6.1 \leq \mathrm{FBG}<7.0 \mathrm{mmol} / \mathrm{L}$ and $7.8 \leq \mathrm{PBG}<11.1 \mathrm{mmol} / \mathrm{L})$, the level of eGFR $\left(\geq 90 \mathrm{~mL} / \mathrm{min}\right.$ per $1.73 \mathrm{~m}^{2}$ and $<90 \mathrm{~mL} / \mathrm{min}$ per $\left.1.73 \mathrm{~m}^{2}\right)$. Potential interactions of VAl quartiles and strata variables were assessed in the logistical regression analysis. In order to further reduce the potential bias and evaluate the association, logistic regression model adjusted for the propensity score was also built to provide an association between VAl quartiles and increased albuminuria. $P$ values $<0.05$ (two-sided) were considered statistically significant.

\section{Results}

\section{Demographic and clinical characteristics of the study population}


After propensity score matching, a total of 24871 prediabetic participants with a median age (Q1-Q3) of 56.15 (51.13-61.74) were enrolled in this study, including 7014 (28.20\%) men and 17857 (71.80\%) women (Table S1). Table 1 presented the basic characteristics of the study population before and after propensity score matching according to UACR range (UACR $<30$ or $\geq 30 \mathrm{mg} / \mathrm{g}$ ). Participants with increased UACR had increased VAI, age, BMI, SBP, DBP, ALT, AST, GGT, TG, FBG, PBG, HbA1c, WC, and decreased eGFR. The prevalence of CVD events was higher in the participants with increased UACR (578 (2.61\%) vs 95 (3.53\%), $p=0.005)$. 
Table 1

Characteristics of study population by UACR category before and after propensity score matching.

\section{before propensity score matching}

\begin{tabular}{|c|c|c|c|c|c|c|}
\hline Variable & $\begin{array}{l}\text { UACR < } \\
30 \mathrm{mg} / \mathrm{g} \\
(\mathrm{n}=25108)\end{array}$ & $\begin{array}{l}\text { UACR } \geq \\
30 \mathrm{mg} / \mathrm{g} \\
(\mathrm{n}=2963)\end{array}$ & $\begin{array}{l}\mathrm{P}- \\
\text { value }\end{array}$ & $\begin{array}{l}\text { UACR < } \\
30 \mathrm{mg} / \mathrm{g} \\
(\mathrm{n}=22180)\end{array}$ & $\begin{array}{l}\text { UACR } \geq \\
30 \mathrm{mg} / \mathrm{g} \\
(\mathrm{n}=2691)\end{array}$ & $\begin{array}{l}\mathrm{P} \text { - } \\
\text { value }\end{array}$ \\
\hline Age, years & $\begin{array}{l}55.87 \\
(50.68- \\
61.33)\end{array}$ & $\begin{array}{l}58.73 \\
(52.97- \\
66.24)\end{array}$ & $<.001$ & $\begin{array}{l}55.92(50.95- \\
61.28)\end{array}$ & $\begin{array}{l}58.67 \\
(53.04- \\
66.16)\end{array}$ & $\begin{array}{l}< \\
0.001\end{array}$ \\
\hline $\mathrm{BMI}, \mathrm{kg} / \mathrm{m} 2$ & $\begin{array}{l}23.73(21.67- \\
26.00)\end{array}$ & $\begin{array}{l}23.94 \\
(21.83- \\
26.37)\end{array}$ & $\begin{array}{l}< \\
0.001\end{array}$ & $\begin{array}{l}23.81(21.76- \\
26.07)\end{array}$ & $\begin{array}{l}24.00 \\
(21.88- \\
26.37)\end{array}$ & 0.005 \\
\hline $\mathrm{SBP}, \mathrm{mmHg}$ & $\begin{array}{l}124.00 \\
(113.00- \\
138.00)\end{array}$ & $\begin{array}{l}130.00 \\
(117.00- \\
147.00)\end{array}$ & $<0.001$ & $\begin{array}{l}125.00(114.00- \\
139.00)\end{array}$ & $\begin{array}{l}131.00 \\
(117.00- \\
148.00)\end{array}$ & $\begin{array}{l}<.001 \\
0.00\end{array}$ \\
\hline $\mathrm{DBP}, \mathrm{mmHg}$ & $\begin{array}{l}75.00 \\
(69.00- \\
82.00)\end{array}$ & $\begin{array}{l}77.00 \\
(70.00- \\
85.00)\end{array}$ & $<.001$ & $\begin{array}{l}75.00(69.00- \\
82.00)\end{array}$ & $\begin{array}{l}77.00 \\
(70.00- \\
85.00)\end{array}$ & $\begin{array}{l}< \\
0.001\end{array}$ \\
\hline ALT, U/L & $\begin{array}{l}14.00 \\
(11.00- \\
20.00)\end{array}$ & $\begin{array}{l}15.00 \\
(11.00- \\
21.00)\end{array}$ & 0.042 & $\begin{array}{l}14.00(11.00- \\
20.00)\end{array}$ & $\begin{array}{l}15.00 \\
(11.00- \\
21.00)\end{array}$ & $\begin{array}{l}<.001 \\
0.00\end{array}$ \\
\hline AST, U/L & $\begin{array}{l}20.00 \\
(17.00- \\
24.00)\end{array}$ & $\begin{array}{l}21.00 \\
(17.00- \\
25.00)\end{array}$ & $<0.001$ & $\begin{array}{l}20.00(17.00- \\
24.00)\end{array}$ & $\begin{array}{l}21.00 \\
(18.00- \\
26.00)\end{array}$ & $\begin{array}{l}<.001 \\
0.00\end{array}$ \\
\hline GGT, U/L & $\begin{array}{l}19.00 \\
(14.00- \\
29.00)\end{array}$ & $\begin{array}{l}21.00 \\
(14.00- \\
30.00)\end{array}$ & 0.029 & $\begin{array}{l}19.00(14.00- \\
29.00)\end{array}$ & $\begin{array}{l}20.00 \\
(14.00- \\
31.00)\end{array}$ & 0.005 \\
\hline $\mathrm{TG}, \mathrm{mmol} / \mathrm{L}$ & $\begin{array}{l}1.24(0.90- \\
1.77)\end{array}$ & $\begin{array}{l}1.41(1.00- \\
2.00)\end{array}$ & $<.001$ & $\begin{array}{l}1.27(0.92- \\
1.81)\end{array}$ & $\begin{array}{l}1.43(1.03- \\
2.04)\end{array}$ & $\begin{array}{l}<.001 \\
0.00\end{array}$ \\
\hline $\mathrm{TC}, \mathrm{mmol} / \mathrm{L}$ & $\begin{array}{l}5.01(4.28- \\
5.75)\end{array}$ & $\begin{array}{l}4.88(4.19- \\
5.60)\end{array}$ & $<.001$ & $\begin{array}{l}5.05(4.33- \\
5.78)\end{array}$ & $\begin{array}{l}4.93(4.24- \\
5.64)\end{array}$ & $\begin{array}{l}< \\
0.001\end{array}$ \\
\hline $\mathrm{HDL}, \mathrm{mmol} / \mathrm{L}$ & $\begin{array}{l}1.32(1.11- \\
1.56)\end{array}$ & $\begin{array}{l}1.28(1.08- \\
1.49)\end{array}$ & $<.001$ & $\begin{array}{l}1.33(1.12- \\
1.56)\end{array}$ & $\begin{array}{l}1.29(1.09- \\
1.50)\end{array}$ & $\dot{0.001}$ \\
\hline
\end{tabular}

Data were mean \pm SD or median (Q1-Q3) for non-normal distribution of variables or numbers $(\%)$ for categorical variables

BMI: body mass index; SBP: systolic blood pressure; DBP: diastolic blood pressure; FBG: fasting plasma glucose; PBG: 2 h post-load blood glucose; HbA1c: glycosylated hemoglobin; ALT: alanine transferase; AST: aspartate transferase; GGT: gamma-glutamyl transferase; TG: triglyceride; TC: high cholesterol; LDL: low-density lipoprotein cholesterol; HDL: high-density lipoprotein cholesterol; eGFR: lower estimated glomerular filtration rate; CVD: cardiovascular disease; WC: waist circumstance; UACR: urinary albumin to creatinine ratio 


\begin{tabular}{|c|c|c|c|c|c|c|}
\hline \multirow[b]{2}{*}{$\mathrm{LDL}, \mathrm{mmol} / \mathrm{L}$} & \multicolumn{3}{|c|}{ before propensity score matching } & \multicolumn{3}{|c|}{ after propensity score matching } \\
\hline & $\begin{array}{l}2.91(2.34- \\
3.52)\end{array}$ & $\begin{array}{l}2.77(2.21- \\
3.37)\end{array}$ & $<.001$ & $\begin{array}{l}2.94(2.37- \\
3.55)\end{array}$ & $\begin{array}{l}2.80(2.26- \\
3.39)\end{array}$ & $<.001$ \\
\hline $\mathrm{FBG}, \mathrm{mmol} / \mathrm{L}$ & $\begin{array}{l}5.40(5.02- \\
5.80)\end{array}$ & $\begin{array}{l}5.46(5.06- \\
5.94)\end{array}$ & $<$ & $\begin{array}{l}5.40(5.03- \\
5.81)\end{array}$ & $\begin{array}{l}5.46(5.05- \\
5.94)\end{array}$ & $<.001$ \\
\hline PBG, mmol/L & $\begin{array}{l}6.85(5.78- \\
8.29)\end{array}$ & $\begin{array}{l}7.30(5.98- \\
8.90)\end{array}$ & $<.001$ & $\begin{array}{l}6.83(5.76- \\
8.28)\end{array}$ & $\begin{array}{l}7.30(5.97- \\
8.90)\end{array}$ & $\begin{array}{l}< \\
0.001\end{array}$ \\
\hline $\mathrm{HbA} 1 \mathrm{c}, \%$ & $\begin{array}{l}5.80(5.50- \\
6.10)\end{array}$ & $\begin{array}{l}5.90(5.60- \\
6.10)\end{array}$ & $<.001$ & $\begin{array}{l}5.80(5.50- \\
6.10)\end{array}$ & $\begin{array}{l}5.90(5.60- \\
6.10)\end{array}$ & $\begin{array}{l}< \\
0.001\end{array}$ \\
\hline $\begin{array}{l}\text { eGFR, } \mathrm{ml} / \mathrm{min} \\
\text { per } 1.73 \mathrm{~m} 2\end{array}$ & $\begin{array}{l}96.51(92.63- \\
100.23)\end{array}$ & $\begin{array}{l}94.64 \\
(89.65- \\
98.79)\end{array}$ & $\begin{array}{l}<.001 \\
0.001\end{array}$ & $\begin{array}{l}96.48(92.65- \\
100.05)\end{array}$ & $\begin{array}{l}94.67 \\
(89.70- \\
98.73)\end{array}$ & $\begin{array}{l}< \\
0.001\end{array}$ \\
\hline UACR mg/g & $\begin{array}{l}8.14(5.18- \\
13.42)\end{array}$ & $\begin{array}{l}44.18(35.20- \\
64.56)\end{array}$ & $\begin{array}{l}<.001 \\
0.001\end{array}$ & $\begin{array}{l}8.25(5.22- \\
13.67)\end{array}$ & $\begin{array}{l}44.01 \\
(35.13- \\
63.97)\end{array}$ & $\begin{array}{l}< \\
0.001\end{array}$ \\
\hline $\mathrm{WC}, \mathrm{cm}$ & $\begin{array}{l}84.00 \\
(77.00- \\
90.00)\end{array}$ & $\begin{array}{l}85.00 \\
(78.30- \\
92.00)\end{array}$ & $\dot{0} 001$ & $\begin{array}{l}84.00(78.00- \\
91.00)\end{array}$ & $\begin{array}{l}85.00 \\
(79.00- \\
92.00)\end{array}$ & $\begin{array}{l}< \\
0.001\end{array}$ \\
\hline $\begin{array}{l}\text { Visceral } \\
\text { adiposity index }\end{array}$ & $\begin{array}{l}1.63(1.07- \\
2.55)\end{array}$ & $\begin{array}{l}1.94(1.30- \\
3.06)\end{array}$ & $<0.001$ & $\begin{array}{l}1.66(1.08- \\
2.59)\end{array}$ & $\begin{array}{l}1.96(1.32- \\
3.07)\end{array}$ & $<.001$ \\
\hline Men, \% & $\begin{array}{l}7238 \\
(28.83 \%)\end{array}$ & $648(21.87 \%)$ & $\begin{array}{l}< \\
0.001\end{array}$ & $6431(29.00 \%)$ & $\begin{array}{l}583 \\
(21.67 \%)\end{array}$ & $<.001$ \\
\hline $\begin{array}{l}\text { Smoking } \\
\text { habits, \% }\end{array}$ & & & 0.007 & & & $\begin{array}{l}< \\
0.001\end{array}$ \\
\hline No & $\begin{array}{l}21320 \\
(84.91 \%)\end{array}$ & $\begin{array}{l}2610 \\
(88.09 \%)\end{array}$ & & $\begin{array}{l}18855 \\
(85.01 \%)\end{array}$ & $\begin{array}{l}2375 \\
(88.26 \%)\end{array}$ & \\
\hline Occasional & $791(3.15 \%)$ & $75(2.53 \%)$ & & $683(3.08 \%)$ & $66(2.45 \%)$ & \\
\hline Regular & $\begin{array}{l}2997 \\
(11.94 \%)\end{array}$ & $278(9.38 \%)$ & & $2642(11.91 \%)$ & $\begin{array}{l}250 \\
(9.29 \%)\end{array}$ & \\
\hline Drinking, $\%$ & & & $<$ & & & $<.001$ \\
\hline
\end{tabular}

Data were mean \pm SD or median (Q1-Q3) for non-normal distribution of variables or numbers (\%) for categorical variables

BMI: body mass index; SBP: systolic blood pressure; DBP: diastolic blood pressure; FBG: fasting plasma glucose; PBG: $2 \mathrm{~h}$ post-load blood glucose; HbA1c: glycosylated hemoglobin; ALT: alanine transferase; AST: aspartate transferase; GGT: gamma-glutamyl transferase; TG: triglyceride; TC: high cholesterol; LDL: low-density lipoprotein cholesterol; HDL: high-density lipoprotein cholesterol; eGFR: lower estimated glomerular filtration rate; CVD: cardiovascular disease; WC: waist circumstance; UACR: urinary albumin to creatinine ratio 


\begin{tabular}{|c|c|c|c|c|}
\hline \multirow[b]{2}{*}{ No } & \multicolumn{2}{|c|}{ before propensity score matching } & \multicolumn{2}{|c|}{ after propensity score matching } \\
\hline & $\begin{array}{l}18291 \\
(72.85 \%)\end{array}$ & $\begin{array}{l}2338 \\
(78.91 \%)\end{array}$ & $\begin{array}{l}16174 \\
(72.92 \%)\end{array}$ & $\begin{array}{l}2121 \\
(78.82 \%)\end{array}$ \\
\hline Occasional & $\begin{array}{l}5212 \\
(20.76 \%)\end{array}$ & $474(16.00 \%)$ & $4575(20.63 \%)$ & $\begin{array}{l}430 \\
(15.98 \%)\end{array}$ \\
\hline Regular & $1605(6.39 \%)$ & $151(5.10 \%)$ & $1431(6.45 \%)$ & $\begin{array}{l}140 \\
(5.20 \%)\end{array}$ \\
\hline $\begin{array}{l}\text { Previous CVD } \\
\text { events, } \%\end{array}$ & & & & 0.005 \\
\hline No & $\begin{array}{l}24463 \\
(97.43 \%)\end{array}$ & $\begin{array}{l}2860 \\
(96.52 \%)\end{array}$ & $\begin{array}{l}21602 \\
(97.39 \%)\end{array}$ & $\begin{array}{l}2596 \\
(96.47 \%)\end{array}$ \\
\hline Yes & $645(2.57 \%)$ & $103(3.48 \%)$ & $578(2.61 \%)$ & $95(3.53 \%)$ \\
\hline \multicolumn{5}{|c|}{$\begin{array}{l}\text { Data were mean } \pm \text { SD or median (Q1-Q3) for non-normal distribution of variables or numbers (\%) for } \\
\text { categorical variables }\end{array}$} \\
\hline \multicolumn{5}{|c|}{$\begin{array}{l}\text { BMI: body mass index; SBP: systolic blood pressure; DBP: diastolic blood pressure; FBG: fasting } \\
\text { plasma glucose; PBG: } 2 \text { h post-load blood glucose; HbA1c: glycosylated hemoglobin; ALT: alanine } \\
\text { transferase; AST: aspartate transferase; GGT: gamma-glutamyl transferase; TG: triglyceride; TC: high } \\
\text { cholesterol; LDL: low-density lipoprotein cholesterol; HDL: high-density lipoprotein cholesterol; eGFR: } \\
\text { lower estimated glomerular filtration rate; CVD: cardiovascular disease; WC: waist circumstance; } \\
\text { UACR: urinary albumin to creatinine ratio }\end{array}$} \\
\hline
\end{tabular}

\section{Factors associated with increased UACR}

Univariate analysis showed that age, BMI, ALT, AST, SBP, DBP, HbA1c were positively correlated with UACR, but eGFR and LDL were negatively correlated with UACR. Also, sex and CVD events were significantly associated with UACR as shown in Table 2. 
Table 2

Univariate analysis of categorical UACR.

\begin{tabular}{|lll|}
\hline Variable & Statistics & $\begin{array}{l}\text { Categorical UACR } \\
\text { OR }(95 \% \mathrm{Cl}), \text { P value }\end{array}$ \\
\hline Age, years & $56.93 \pm 8.84$ & $1.04(1.03,1.04)<0.0001$ \\
\hline Sex & & \\
\hline men & $7014(24.20 \%)$ & 1.0 \\
\hline women & $21380(75.80 \%)$ & $1.48(1.34,1.63)<0.0001$ \\
\hline BMI, kg/m & $24.11 \pm 3.58$ & $1.02(1.00,1.03) 0.0053$ \\
\hline ALT, U/L & $17.33 \pm 12.91$ & $1.00(1.00,1.01) 0.0004$ \\
\hline AST, U/L & $22.13 \pm 11.29$ & $1.01(1.01,1.01)<0.0001$ \\
\hline eGFR, ml/min per 1.73 m² & $95.93 \pm 6.02$ & $0.95(0.94,0.96)<0.0001$ \\
\hline DBP, mm/Hg & $76.27 \pm 10.82$ & $1.02(1.01,1.02)<0.0001$ \\
\hline SBP, mm/Hg & $128.03 \pm 19.59$ & $1.02(1.01,1.02)<0.0001$ \\
\hline HbA1c, \% & $5.82 \pm 0.47$ & $1.46(1.35,1.58)<0.0001$ \\
\hline LDL, mmol/L & $2.98 \pm 0.89$ & $0.83(0.80,0.87)<0.0001$ \\
\hline CVD events & & \\
\hline No & $24198(97.29 \%)$ & 1.0 \\
\hline Yes & $673(2.71 \%)$ & $1.37(1.10,1.71) 0.0054$ \\
\hline ations Of VAl quartiles With increased UACR
\end{tabular}

The median UACR (Q1-Q3) was 6.72 (4.25-11.98), 7.60 (4.59-14.14), 8.35 (5.05-14.60), 8.75 (5.28$16.07)$ in men, and 8.16 (5.19-14.46), 9.35 (5.73-17.78), 10.67 (6.38-20.26), 12.06 (6.91-22.55) in women from the lowest to the highest VAl quartile (Fig. 2).

Table 3 showed the association between VAI quartiles and increased UACR in total population in Model 1Model 5. As shown in Table 3, compared with participants in the first VAl quartile, those in Q2, Q3, Q4 of VAl, respectively, have higher risk of increased UACR in Model 1-4 (P for trend $<0.001$ ). After further adjusting for SBP, DBP, LDL, HbA1c, the association was still significant in Model 5 , indicating the stability of the relationship between VAI quartiles and increased UACR (Q2: OR:1.10, 95\% Cl 0.96-1.25; Q3:

OR:1.16, 95\% Cl 1.01-1.32; Q4: OR:1.26, 95\% Cl 1.10-1.44, P for trend = 0.001). In women, compared with the first quartile, the odds of increased UACR in Q2, Q3, Q4 was significantly higher in Model 1-Model 4, and the relationship was still significant even after further adjusting for SBP, DBP, LDL, HbA1c in Model 5 as shown in Table 4 (Model 5: Q2: OR:1.12, 95\% Cl 0.96-1.31; Q3:OR:1.16, 95\%Cl 0.99-1.36; Q4:OR:1.30, 
$95 \% \mathrm{Cl} 1.11-1.52$, $\mathrm{P}$ for trend $=0.001)$. However, as seen in Table 4, in men, the relationship was attenuated in Model 1-4, and no significant association between VAI quartiles and increased UACR was observed after additionally adjusting for SBP, DBP, LDL, HbA1c.

Table 3

Association between VAl quartiles and UACR in the total population with prediabetes.

\begin{tabular}{|c|c|c|c|c|c|}
\hline \multirow[t]{2}{*}{ Variable } & \multicolumn{5}{|c|}{ VAl quartiles } \\
\hline & Q1 & Q2 & Q3 & Q4 & $\begin{array}{l}P \text { for } \\
\text { trend }\end{array}$ \\
\hline $\begin{array}{l}\text { Model } 1 \\
\text { OR } \\
(95 \% \mathrm{Cl})\end{array}$ & 1.0 & $\begin{array}{l}1.42(1.26,1.61)< \\
0.001\end{array}$ & $\begin{array}{l}1.73(1.53,1.95)< \\
0.001\end{array}$ & $\begin{array}{l}2.15(1.91,2.42)< \\
0.001\end{array}$ & $<0.001$ \\
\hline \multicolumn{6}{|l|}{$P$ value } \\
\hline $\begin{array}{l}\text { Model } 2 \\
\text { OR } \\
(95 \% \mathrm{Cl})\end{array}$ & 1.0 & $\begin{array}{l}1.16(1.02,1.32) \\
0.023\end{array}$ & $\begin{array}{l}1.26(1.11,1.43) \\
0.000\end{array}$ & $\begin{array}{l}1.49(1.31,1.69)< \\
0.001\end{array}$ & $<0.001$ \\
\hline \multicolumn{6}{|l|}{$P$ value } \\
\hline $\begin{array}{l}\text { Model } 3 \\
\text { OR } \\
(95 \% \mathrm{Cl})\end{array}$ & 1.0 & $\begin{array}{l}1.14(1.00,1.30) \\
0.046\end{array}$ & $\begin{array}{l}1.23(1.08,1.40) \\
0.002\end{array}$ & $\begin{array}{l}1.44(1.26,1.64)< \\
0.001\end{array}$ & $<0.001$ \\
\hline \multicolumn{6}{|l|}{$P$ value } \\
\hline $\begin{array}{l}\text { Model } 4 \\
\text { OR } \\
(95 \% \mathrm{Cl})\end{array}$ & 1.0 & $\begin{array}{l}1.14(1.00,1.30) \\
0.042\end{array}$ & $\begin{array}{l}1.23(1.08,1.40) \\
0.002\end{array}$ & $\begin{array}{l}1.44(1.26,1.64)< \\
0.001\end{array}$ & $<0.001$ \\
\hline \multicolumn{6}{|l|}{$P$ value } \\
\hline $\begin{array}{l}\text { Model } 5 \\
\text { OR } \\
(95 \% \mathrm{Cl})\end{array}$ & 1.0 & $\begin{array}{l}1.10(0.96,1.25) \\
0.167\end{array}$ & $\begin{array}{l}1.16(1.01,1.32) \\
0.030\end{array}$ & $\begin{array}{l}1.26(1.10,1.44) \\
0.001\end{array}$ & 0.001 \\
\hline \multicolumn{6}{|l|}{$P$ value } \\
\hline \multicolumn{6}{|c|}{ Model 1: unadjusted; } \\
\hline \multicolumn{6}{|c|}{ Model 2: adjusted for age, sex, centers; } \\
\hline \multicolumn{6}{|c|}{ Model 3: additionally adjusted for BMI, ALT, AST, eGFR based on Model 2; } \\
\hline Model 4: & ition & ly adjusted for smo & , drinking, CVD eve & ased on Model 3; & \\
\hline
\end{tabular}


Table 4

Association between VAl quartiles and UACR by gender in participants with prediabetes.

\begin{tabular}{|c|c|c|c|c|c|}
\hline \multirow[t]{2}{*}{ Variable } & Model 1 & Model 2 & Model 3 & Model 4 & Model 5 \\
\hline & $\begin{array}{l}\text { OR }(95 \% \mathrm{Cl}), \mathrm{P} \\
\text { value }\end{array}$ & $\begin{array}{l}\text { OR }(95 \% \mathrm{Cl}), \mathrm{P} \\
\text { value }\end{array}$ & $\begin{array}{l}\text { OR }(95 \% \mathrm{Cl}), \mathrm{P} \\
\text { value }\end{array}$ & $\begin{array}{l}\text { OR }(95 \% \mathrm{Cl}), \mathrm{P} \\
\text { value }\end{array}$ & $\begin{array}{l}\text { OR }(95 \% \mathrm{Cl}), \mathrm{P} \\
\text { value }\end{array}$ \\
\hline \multicolumn{6}{|l|}{ Men, } \\
\hline \multicolumn{6}{|l|}{$\begin{array}{l}\text { VAl } \\
\text { quartiles }\end{array}$} \\
\hline Q1 & 1.0 & 1.0 & 1.0 & 1.0 & 1.0 \\
\hline Q2 & $\begin{array}{l}1.22(0.97 \\
1.54) 0.095\end{array}$ & $\begin{array}{l}1.16(0.91 \\
1.47) 0.224\end{array}$ & $\begin{array}{l}1.11(0.87 \\
1.42) 0.391\end{array}$ & $\begin{array}{l}1.11(0.87 \\
1.42) 0.382\end{array}$ & $\begin{array}{l}1.05(0.82 \\
1.34) 0.707\end{array}$ \\
\hline Q3 & $\begin{array}{l}1.45(1.15 \\
1.84) 0.002\end{array}$ & $\begin{array}{l}1.37(1.07 \\
1.74) 0.012\end{array}$ & $\begin{array}{l}1.29(1.00 \\
1.66) 0.046\end{array}$ & $\begin{array}{l}1.29(1.00 \\
1.66) 0.049\end{array}$ & $\begin{array}{l}1.19(0.92 \\
1.53) 0.195\end{array}$ \\
\hline Q4 & $\begin{array}{l}1.49(1.17 \\
1.90) 0.002\end{array}$ & $\begin{array}{l}1.51(1.17 \\
1.94) 0.001\end{array}$ & $\begin{array}{l}1.36(1.04 \\
1.77) 0.023\end{array}$ & $\begin{array}{l}1.35(1.04 \\
1.76) 0.026\end{array}$ & $\begin{array}{l}1.15(0.88 \\
1.52) 0.306\end{array}$ \\
\hline $\begin{array}{l}P \text { for } \\
\text { trend }\end{array}$ & 0.001 & 0.012 & 0.020 & 0.024 & 0.2860 \\
\hline \multicolumn{6}{|l|}{ Women } \\
\hline \multicolumn{6}{|l|}{$\begin{array}{l}\text { VAl } \\
\text { quartiles }\end{array}$} \\
\hline Q1 & 1.0 & 1.0 & 1.0 & 1.0 & 1.0 \\
\hline Q2 & $\begin{array}{l}1.48(1.27 \\
1.71)<0.001\end{array}$ & $\begin{array}{l}1.17(1.00 \\
1.36) 0.045\end{array}$ & $\begin{array}{l}1.15(0.99 \\
1.34) 0.072\end{array}$ & $\begin{array}{l}1.15(0.99 \\
1.35) 0.069\end{array}$ & $\begin{array}{l}1.12(0.96 \\
1.31) 0.155\end{array}$ \\
\hline Q3 & $\begin{array}{l}1.78(1.54 \\
2.06)<0.001\end{array}$ & $\begin{array}{l}1.24(1.07 \\
1.44) 0.005\end{array}$ & $\begin{array}{l}1.22(1.05 \\
1.42) 0.012\end{array}$ & $\begin{array}{l}1.22(1.05 \\
1.42) 0.011\end{array}$ & $\begin{array}{l}1.16(0.99 \\
1.36) 0.062\end{array}$ \\
\hline Q4 & $\begin{array}{l}2.29(1.99 \\
2.64)<0.001\end{array}$ & $\begin{array}{l}1.49(1.28 \\
1.73)<0.001\end{array}$ & $\begin{array}{l}1.45(1.25 \\
1.69)<0.001\end{array}$ & $\begin{array}{l}1.45(1.25 \\
1.70)<0.001\end{array}$ & $\begin{array}{l}1.30(1.11 \\
1.52) 0.001\end{array}$ \\
\hline $\begin{array}{l}P \text { for } \\
\text { trend }\end{array}$ & $<0.001$ & $<0.001$ & $<0.001$ & $<0.001$ & 0.001 \\
\hline \multicolumn{6}{|c|}{ Model 1: unadjusted; } \\
\hline \multicolumn{6}{|c|}{ Model 2: adjusted for age, centers; } \\
\hline \multicolumn{6}{|c|}{ Model 3: additionally adjusted for BMI, ALT, AST, eGFR based on Model 2; } \\
\hline \multicolumn{6}{|c|}{ Model 4: additionally adjusted for smoking, drinking, CVD events based on Model 3; } \\
\hline Model 5 & itionally adj & for SBP, DBP, L & $\mathrm{HbA} 1_{\mathrm{C}}$ based & odel 4. & \\
\hline
\end{tabular}




\section{Associations of VAl quartiles with increased UACR in stratified analysis}

Stratified analysis was performed to further confirm the stability of the relationship between VAI quartiles and increased UACR. Table 5 showed that the associations between VAI quartiles and increased UACR were not consistent in subgroups. Compared with the first quartile of VAl, younger participants (age $₫ 60$ years) in Q2, Q3,Q4 were more likely to have increased UACR. However, no significant association was found in older participants (age $\geq 60$ years). Significant association of VAl quartiles with increased UACR was also observed in participants who were overweight or obese $(24 \leq \mathrm{BMI}<28$ or $\mathrm{BMI} \geq$ $\left.28 \mathrm{~kg} / \mathrm{m}^{2}\right)$, with high-normal blood pressure or hypertension ( $120 \leq \mathrm{SBP}<140$ and/or $80 \leq \mathrm{DBP}<90$ or $\mathrm{SBP} \geq 140$ or $\mathrm{DBP} \geq 90 \mathrm{mmHg}$ ). In order to further explore the association of VAl quartiles with increased UACR in prediabetic participants, participants were divided into IFG, IGT and IFG + IGT subgroups. As seen in Table 5, significant association was found in IFG and IGT groups (IFG: $5.6 \leq \mathrm{FBG}<7.0 \mathrm{mmol} / \mathrm{L}$ and $\mathrm{PBG}<7.8 \mathrm{mmol} / \mathrm{L}, \mathrm{IGT}: \mathrm{FBG}<7.0 \mathrm{mmol} / \mathrm{L}$ and $7.8 \leq \mathrm{PBG}<11.1 \mathrm{mmol} / \mathrm{L}$ ). Compared with participants in the first quartile, those in the higher quartiles of VAl had the most significant association with increased UACR. Moreover, to better investigate the association of VAI quartiles with increased UACR, stratified analysis was performed based on different level of eGFR. Similar results were observed in different level of eGFR. When participant with normal eGFR (eGFR $\geq 90 \mathrm{ml} / \mathrm{min}$ per $1.73 \mathrm{~m}^{2}$ ), the odds of having increased UACR gradually elevated from the lowest to highest quartiles of VAI. However, there was no significant relationship between VAI quartiles and UACR among participants with lower eGFR (eGFR $₫ 90 \mathrm{ml} / \mathrm{min}$ per $1.73 \mathrm{~m}^{2}$ ). Interactions of VAl with stratified variables were not found in stratifications. 
Table 5

Association between VAI quartiles and UACR in different participants with prediabetes.

Variable

\begin{tabular}{|c|c|c|c|c|c|c|}
\hline & Q1 & Q2 & Q3 & Q4 & & \\
\hline & $\begin{array}{l}\text { OR } \\
\text { (95\%Cl), } \\
\text { P value }\end{array}$ & $\begin{array}{l}\text { OR }(95 \% \mathrm{Cl}) \\
\text { P value }\end{array}$ & $\begin{array}{l}\text { OR } \\
\text { (95\%Cl), P } \\
\text { value }\end{array}$ & $\begin{array}{l}\mathrm{OR} \\
(95 \% \mathrm{Cl}), \mathrm{P} \\
\text { value }\end{array}$ & $\begin{array}{l}P \text { for } \\
\text { trend }\end{array}$ & $\begin{array}{l}\text { P for } \\
\text { interaction }\end{array}$ \\
\hline Age, years & & & & & & 0.670 \\
\hline$<60$ & 1.0 & $\begin{array}{l}1.05(0.89 \\
1.25) 0.547\end{array}$ & $\begin{array}{l}1.13(0.96 \\
1.35) 0.150\end{array}$ & $\begin{array}{l}1.28(1.07 \\
1.52) 0.006\end{array}$ & 0.002 & \\
\hline$\geq 60$ & 1.0 & $\begin{array}{l}1.16(0.94 \\
1.43) 0.165\end{array}$ & $\begin{array}{l}1.19(0.96 \\
1.47) 0.112\end{array}$ & $\begin{array}{l}1.22(0.99 \\
1.51) 0.067\end{array}$ & 0.158 & \\
\hline $\mathrm{BMI}, \mathrm{kg} / \mathrm{m}^{2}$ & & & & & & 0.401 \\
\hline$<18.5$ & 1.0 & $\begin{array}{l}0.86(0.47 \\
1.57) 0.630\end{array}$ & $\begin{array}{l}0.67(0.30 \\
1.47) 0.317\end{array}$ & $\begin{array}{l}0.67(0.17 \\
2.56) 0.556\end{array}$ & 0.336 & \\
\hline $18.5 \leq \mathrm{BMI}<24$ & 1.0 & $\begin{array}{l}1.12(0.94 \\
1.33) 0.208\end{array}$ & $\begin{array}{l}1.19(0.99 \\
1.42) 0.066\end{array}$ & $\begin{array}{l}1.18(0.97 \\
1.44) 0.094\end{array}$ & 0.139 & \\
\hline $24 \leq \mathrm{BMI}<28$ & 1.0 & $\begin{array}{l}1.20(0.93 \\
1.54) 0.167\end{array}$ & $\begin{array}{l}1.16(0.90 \\
1.49) 0.245\end{array}$ & $\begin{array}{l}1.39(1.09 \\
1.77) 0.009\end{array}$ & 0.008 & \\
\hline$\geq 28$ & 1.0 & $\begin{array}{l}0.89(0.55 \\
1.45) 0.648\end{array}$ & $\begin{array}{l}1.34(0.85 \\
2.12) 0.200\end{array}$ & $\begin{array}{l}1.37(0.88 \\
2.14) 0.166\end{array}$ & 0.034 & \\
\hline $\begin{array}{l}\text { Blood pressure, } \\
\mathrm{mmHg}\end{array}$ & & & & & & 0.520 \\
\hline $\begin{array}{l}\mathrm{SBP}<120 \text { and } \mathrm{DBP} \\
<80\end{array}$ & 1.0 & $\begin{array}{l}1.04(0.83 \\
1.30) 0.751\end{array}$ & $\begin{array}{l}0.95(0.75 \\
1.21) 0.700\end{array}$ & $\begin{array}{l}1.09(0.85 \\
1.39) 0.514\end{array}$ & 0.552 & \\
\hline $\begin{array}{l}120 \leq \mathrm{SBP}<140 \\
\text { and/or } 80 \leq \mathrm{DBP}< \\
90\end{array}$ & 1.0 & $\begin{array}{l}1.11(0.91 \\
1.35) 0.321\end{array}$ & $\begin{array}{l}1.29(1.06 \\
1.56) 0.013\end{array}$ & $\begin{array}{l}1.31(1.07 \\
1.60) 0.008\end{array}$ & 0.009 & \\
\hline $\begin{array}{l}\mathrm{SBP} \geq 140 \text { or } \mathrm{DBP} \\
\geq 90\end{array}$ & 1.0 & $\begin{array}{l}1.14(0.86 \\
1.52) \\
0.3689\end{array}$ & $\begin{array}{l}1.19(0.89 \\
1.58) 0.244\end{array}$ & $\begin{array}{l}1.38(1.04 \\
1.83) 0.025\end{array}$ & 0.022 & \\
\hline $\begin{array}{l}\text { Prediabetes status, } \\
\mathrm{mmol} / \mathrm{L}\end{array}$ & & & & & & 0.244 \\
\hline IFG & 1.0 & $\begin{array}{l}1.30(0.71 \\
2.39) 0.399\end{array}$ & $\begin{array}{l}2.16(1.20 \\
3.91) 0.011\end{array}$ & $\begin{array}{l}2.00(1.10 \\
3.62) 0.023\end{array}$ & 0.027 & \\
\hline
\end{tabular}

adjusted for age, sex, centers, BMI, ALT, AST, eGFR, smoking, drinking, CVD events, SBP, DBP, LDL, $\mathrm{HbA} 1_{\mathrm{C}}$. 


\begin{tabular}{|c|c|c|c|c|c|}
\hline Variable & VAl & & & & \\
\hline IGT & 1.0 & $\begin{array}{l}1.10(0.95 \\
1.26) 0.205\end{array}$ & $\begin{array}{l}1.15(0.99 \\
1.32) 0.065\end{array}$ & $\begin{array}{l}1.21(1.04 \\
1.40) 0.012\end{array}$ & 0.018 \\
\hline $\mathrm{IFG}+\mathrm{IGT}$ & 1.0 & $\begin{array}{l}0.90(0.58 \\
1.39) 0.622\end{array}$ & $\begin{array}{l}0.85(0.56 \\
1.30) 0.457\end{array}$ & $\begin{array}{l}1.06(0.71 \\
1.59) 0.765\end{array}$ & 0.271 \\
\hline $\begin{array}{l}\text { eGFR, } \mathrm{ml} / \mathrm{min} \text { per } \\
1.73 \mathrm{~m}^{2}\end{array}$ & & & & & 0.561 \\
\hline$<90$ & 1.0 & $\begin{array}{l}1.25(0.95 \\
1.63) 0.107\end{array}$ & $\begin{array}{l}1.36(1.04 \\
1.79) 0.027\end{array}$ & $\begin{array}{l}1.35(1.02 \\
1.80) 0.035\end{array}$ & 0.103 \\
\hline$\geq 90$ & 1.0 & $\begin{array}{l}1.05(0.90 \\
1.22) 0.518\end{array}$ & $\begin{array}{l}1.11(0.95 \\
1.29) 0.195\end{array}$ & $\begin{array}{l}1.22(1.05 \\
1.42) 0.010\end{array}$ & 0.005 \\
\hline
\end{tabular}

Importantly, to provide the association of VAI quartiles with increased UACR in a robust way, the combination of propensity matching score and multivariable logistic regression model was used to analyze the association. Logistic regression model adjusted for the propensity matching score was built to investigate the relationship between VAI quartiles and increased UACR in total population and subgroups and the results were consistent, which were presented in supplementary materials (Table S2Table S4).

\section{Discussion}

\section{Main findings}

In the present study, we found that an elevated VAI was positively associated with UACR in the population with prediabetes and a significant sex difference was observed. The significant association between VAl and increased UACR was only revealed in prediabetic women, whereas such association was no longer significant in prediabetic men after controlling for the potential risk factors. Importantly, after further adjusting for blood pressure, $\mathrm{HbA} 1 \mathrm{c}$, and LDL, the association was weakened, suggesting that abnormal blood pressure, glucose and lipid metabolism would increase the risk of albuminuria in people with prediabetes. Furthermore, stratified analysis revealed that participants with higher VAI were more likely to have increased UACR than those with lower VAl, especially in subjects who were young, overweight or obese, with blood pressure and blood glucose abnormalities, as well as normal values of eGFR. To best of our current knowledge, this is the first multicenter and the largest sample study to investigate the association between VAl and increased UACR in the Chinese population with prediabetes. Therefore, early prevention and intervention are vital for albuminuria, and modification of the abnormal fat distribution may contribute to the early detection and prevention of adverse outcomes, especially in people with obesity, blood pressure and blood glucose abnormalities. 


\section{Visceral obesity and CVD}

As we all know, obesity contributes to hyperglycemia, hypertension, insulin resistance (IR), and is also associated with higher CVD risk. It has been reported that visceral obesity rather than general obesity was closely associated with higher CVD risk ${ }^{[19]}$. According to the International Diabetes Federation, the distribution of visceral obesity can be accurately assessed by magnetic resonance imaging (MRI) and computed tomography (CT), which is considered as the gold standard. However, these measures are timeconsuming, costly, and risky due to radiation exposure, so they are not routinely available, especially in epidemiological research. Therefore, VAI has been proposed as an emerging surrogate, which is an effective, convenient and routinely applicable indicator of visceral fat distribution and dysfunction ${ }^{[8]}$. A prospective study of 3042 adults in Europe demonstrated a significant association between VAI and CVD risk. In this study, a higher level of VAI was independently related with the increased 10-year CVD risk in men ${ }^{[20]}$. In addition, a cross-sectional study conducted in Germany confirmed that VAl is a simple and effective tool to identify CVD risk ${ }^{[21]}$. The study included 731 adults who were free of CVD but were at high risk of T2DM, and found that VAl was associated with subclinical atherosclerosis independent of other cardiometabolic risk factors. Similar results were also found in another study. It has been reported that visceral obesity was positively related with arterial inflammation and people with increased visceral obesity were at higher risk for CVD events ${ }^{[21]}$.

\section{Albuminuria and CVD}

In univariate analysis of our study, we found that CVD was significantly associated with increased albuminuria. Albuminuria has been recognized as a significant indicator of generalized atherosclerosis, because of its association with atherosclerotic risk factors and microvascular endothelial dysfunction ${ }^{\text {[22] }}$. Data from population-based studies showed that albuminuria is related with a higher risk for CVD and cardiovascular mortality ${ }^{[6,23]}$. Similar results were found in nondiabetic and normotensive people ${ }^{[24]}$. A prospective study of 2484 white subjects aged over 50 years reported that albuminuria is associated with 3.22-fold, 1.38-fold increased risk of cardiovascular mortality in all and nondiabetic subjects after adjusting for a wide spectrum of risk factors. And the risk is markedly higher (5.68-fold) in diabetic subjects $^{[25]}$. The relationship was also observed in the general population. The PREVEND study including 40548 participants indicated that a two-fold increment in albuminuria conferred a 1.29-fold increased risk of cardiovascular death ${ }^{[26]}$. Additionally, an association has also been reported between albuminuria, myocardial ischemia, stroke and peripheral vascular diseases in several studies ${ }^{[27-29]}$. The above evidence could support our findings. The presence of albumin in the urine may be generated due to vascular damage, suggesting systemic endothelia dysfunction. Therefore, early identification of warning indicators for albuminuria is of great significance and could contribute to reduce the risk of CVD.

\section{The relationship between visceral obesity and albuminuria}

Previous studies have reported that visceral obesity was significantly associated with albuminuria. In a cross-sectional study of 208 adults with T2DM, visceral obesity presented a significant association with 
$\mathrm{UACR}^{[30]}$. Similarly, another 4-year follow-up study including 2393 participants observed that participants with the greater increase in visceral fat mass had a higher risk for albuminuria ${ }^{[31]}$. However, these studies are less reliable, due to the small sample size. Sun et al. also investigated a positive relationship between visceral obesity and albuminuria ${ }^{[15]}$. However, in their study, participants with renal or liver diseases were not excluded, possibly resulting in residual confounding effects. Importantly, few studies investigated the relationship between VAI and albuminuria in prediabetic individuals who are most likely to develop T2DM and have more potential cardiovascular risk factors.

In our study, 24871 participants with prediabetes were included and we found that higher visceral obesity evaluated by VAI was independently associated with the increased risk of albuminuria. The results were consistent with previous studies. Furthermore, a sex difference was detected in our study, which may be partly explained by the gender-specific hormone in the elderly people. Elderly women who are in the perimenopausal or postmenopausal stage, are more prone to fat redistribution, owing to hormones alteration. The decreased estrogen levels after menopause is closely associated with increased adiposity and visceral fat accumulation ${ }^{[32]}$. This could be supported by the studies from Ryan et al. ${ }^{[33]}$. Therefore, women probably have the more accumulation of visceral fat ${ }^{[34]}$, leading to a greater risk for the progression of inflammation and kidney damage.

Notably, we found that the association between VAl and albuminuria was attenuated after further adjusting for the $\mathrm{HbA} 1 \mathrm{c}$, blood pressure and $\mathrm{LDL}$ in model 5 , suggesting that metabolism abnormalities may increase the risk of albuminuria. Many cross-sectional and prospective studies have reported significant relationships between albuminuria, hypertension, diabetes and dyslipidemia, which could partly explain the results. In this study, we also analyzed the relationship between VAI and albuminuria in different stratification. Significant associations were detected in people who were overweight, obese and with abnormal blood pressure and blood glucose metabolisms as well as normal eGFR. Thus, not only modification of the distribution of visceral fat, but also the early detection and intervention of established risk factors in such people are of great importance. Interestingly, we found that the association was only significant in younger participants rather than older ones in this study. However, no interaction of VAI and age in UACR groups was observed. The speculation is that older participants were inclined to keep a healthy diet and have a better compliance, possibly contributing to the favorable effect of albuminuria prevention.

It is well established that WC has been considered as a major clinical parameter of visceral adiposity distribution. However, WC alone is unable to distinguish visceral adiposity from subcutaneous adiposity ${ }^{[35]}$. In this considerable drawback, VAl has been confirmed as a useful and valid indicator of visceral adiposity distribution and dysfunction, which combined the measurement of anthropometric indices (WC and BMI) with the assessment of lipid metabolism (TG and HDL). Numerous studies have supported that regional visceral fat deposition was a key adiposity phenotype associated with the development of CVD, T2DM, hypertension, CKD and metabolic complications. UACR as a known indicator of kidney damage is well predictive of atherogenic state. Accordingly, population-based studies pointed 
out that UACR is valuable in predicting clinical cardiovascular outcomes ${ }^{[6,23]}$. By using VAl to evaluate visceral obesity, the relationship between visceral obesity and UACR has been reported in some studies ${ }^{[15}$, ${ }^{30,31]}$. Most studies on this issue, however, have failed to investigate the relationship in prediabetic individuals who are at high risk for the development of T2DM and metabolic complications. People with prediabetes may not be aware of glycemic control and the improvement of fat distribution for a long time, resulting in the progression of T2DM and the incidence of diabetic nephropathy. In this regard, our study provided more evidence to corroborate the association of visceral adiposity assessed by VAI and albuminuria in prediabetic population and indicate the value of VAl as a simple, reliable and efficient screening tool for metabolic complications. Improving the distribution and deposition of visceral fat rather than just weight loss should be proposed to reduce related-CVD risk in clinical practice.

\section{Limitation}

This multicenter study has a large sample size, a comprehensive adjustment for major traditional risk factors, and represented the prediabetic people from different regions across China. However, some limitations should be noted. Firstly, considering all participants from China, our study may not be completely representative of other ethnic populations. More studies of other ethnic groups are expected to confirm the relationship. Secondly, due to the nature of this cross-sectional study, the causal relationship between VAI and albuminuria cannot be determined. Thus, the association of VAI with albuminuria should be further explored in follow-up studies. Thirdly, in spite of excluding the participants using ACEI/ARB, lipid-lowering, hypoglycemic and other hypertensive drugs in our study, there are still possibility that other medications may partly affect the association of VAI with albuminuria. Fourthly, our study did not assess visceral fat tissue precisely by MRI or CT, because these measurements are costly and inconvenient in large-scale population studies. However, the close association of VAl and visceral fat tissue has been confirmed in previous studies ${ }^{[8,36]}$.In fact, what we want to emphasize is that the value of VAl as a reliable, simple and efficient screening tool to identify increased risk of albuminuria in population with prediabetes.

\section{Conclusion}

The present study observed that increased level of VAI was significantly associated with increased risk of albuminuria in prediabetic individuals. People with elevated VAl were at higher risk for albuminuria, especially in subjects who were young, overweight or obese, with blood pressure and blood glucose abnormalities, as well as eGFR $\geq 90 \mathrm{~mL} / \mathrm{min}$ per $1.73 \mathrm{~m}^{2}$. The findings provided new insights into the value of VAI as a reliable and efficient screening tool for the identification of prediabetic people at high risk of albuminuria to further reduce the incidence of unfavorable cardiovascular outcomes in routine clinical practice. Considering the correlation of visceral obesity with albuminuria and CVD, we should pay more attention to prediabetic patients with regional visceral fat deposition and take effective methods to guide them to improve the distribution of visceral adiposity instead of just losing weight. 


\section{Abbreviations}

UACR

urinary albumin-creatinine ratio; T2DM:type 2 diabetes mellitus; CVD:cardiovascular diseases; CKD:chronic kidney diseases; VAl:visceral adiposity index; WC:waist circumference; TG:triglycerides; HDL:high density lipoprotein; BMI:body mass index; FPG:fasting plasma glucose; PBG:2 h post-load blood glucose; HbA1c:glycosylated hemoglobin; TC:total cholesterol; LDL:Iow density lipoprotein cholesterol; GGT:gamma-glutamyl transferase; AST:aspartate aminotransferase; ALT:alanine aminotransferase; Cr:creatinine; CKD-EPI:Chronic Kidney Disease Epidemiology Collaboration; eGFR:estimated glomerular filtration rate; IFG:impaired fasting glucose; IGT:impaired glucose tolerance; OR:odds ratio; Cl:confidence intervals; SBP:systolic blood pressure; DBP:diastolic blood pressure; IR:insulin resistance; MRI:magnetic resonance imaging; CT:computed tomography.

\section{Declarations}

\section{Availability of data and materials}

The datasets used to support this study are not freely available due to participants' privacy protection.

\section{Acknowledgements}

We would like to thank the participants in this study.

\section{Competing interests:}

The authors declare no competing interests.

\section{Consent for publication:}

Not applicable.

\section{Ethics approval and consent to participate:}

The study protocol was approved by the Committee on Human Research at RuiJin Hospital affiliated with the School of Medicine, Shanghai Jiao Tong University. Informed consents were provided by all participants before data collection.

\section{Funding:}

The study is supported by the Chinese Society of Endocrinology, the Key Laboratory for Endocrine and Metabolic Diseases of Ministry of Health (1994DP131044), the National Key New Drug Creation and 
Manufacturing Program of Ministry of Science and Technology (2012ZX09303006-001), the National High Technology Research and Development Program of China (863 Program, 2011AA020107), National Science Foundation of China (81300717), National Science and Technology Major Project 288 (2011ZX09307-001-08), the REACTION Study.

\section{Authors' contributions:}

YM and JW contributed to the conception and design of the study. XJ, KC, WY, AW, WW, ZG, X T, LY, QW, $Z L, G Q$ and $L C$ recruited the subjects and supervised the study. JW analyzed the data and wrote the initial draft of the paper. YM and JW, BZ contributed to the writing, reviewing, and revising of the manuscript.

\section{Acknowledgements:}

Not applicable

\section{References}

1. Matsushita $\mathrm{K}$, et al. Association of estimated glomerular filtration rate and albuminuria with all-cause and cardiovascular mortality in general population cohorts: a collaborative meta-analysis. Lancet. 2010;375(9731):2073-81.

2. Astor BC, et al. Lower estimated glomerular filtration rate and higher albuminuria are associated with mortality and end-stage renal disease. A collaborative meta-analysis of kidney disease population cohorts. Kidney Int. 2011;79(12):1331-40.

3. Rifkin DE, et al. Albuminuria, impaired kidney function and cardiovascular outcomes or mortality in the elderly. Nephrol Dial Transplant. 2010;25(5):1560-7.

4. Ninomiya T, et al. Albuminuria and kidney function independently predict cardiovascular and renal outcomes in diabetes. J Am Soc Nephrol. 2009;20(8):1813-21.

5. Li MF, et al. Low-grade albuminuria is associated with early but not late carotid atherosclerotic lesions in community-based patients with type 2 diabetes. Cardiovasc Diabetol. 2013;12:110.

6. Gerstein $\mathrm{HC}$, et al. Albuminuria and risk of cardiovascular events, death, and heart failure in diabetic and nondiabetic individuals. Jama. 2001;286(4):421-6.

7. Garofalo C, et al. A systematic review and meta-analysis suggests obesity predicts onset of chronic kidney disease in the general population. Kidney Int. 2017;91(5):1224-35.

8. Amato MC, et al. Visceral Adiposity Index: a reliable indicator of visceral fat function associated with cardiometabolic risk. Diabetes Care. 2010;33(4):920-2.

9. Bouchi R, et al. High visceral fat with low subcutaneous fat accumulation as a determinant of atherosclerosis in patients with type 2 diabetes. Cardiovasc Diabetol. 2015;14:136. 
10. Fox CS, et al. Abdominal visceral and subcutaneous adipose tissue compartments: association with metabolic risk factors in the Framingham Heart Study. Circulation. 2007;116(1):39-48.

11. Franca AK, et al. Estimated visceral adipose tissue, but not body mass index, is associated with reductions in glomerular filtration rate based on cystatin $C$ in the early stages of chronic kidney disease. Int J Nephrol. 2014;2014:574267.

12. Fiorentino TV. Visceral adiposity index (VAI), a powerful predictor of incident hypertension in prehypertensives. Intern Emerg Med. 2018;13(4):471-3.

13. Wei J, et al., Comparisons of Visceral Adiposity Index, Body Shape Index, Body Mass Index and Waist Circumference and Their Associations with Diabetes Mellitus in Adults. Nutrients, 2019. 11(7).

14. Vassilatou $E$, et al. Visceral adiposity index for the diagnosis of nonalcoholic fatty liver disease in premenopausal women with and without polycystic ovary syndrome. Maturitas. 2018;116:1-7.

15. Sun K, et al. Visceral adiposity index is associated with increased urinary albumin excretion: A population-based study. Clin Nutr. 2019;38(3):1332-8.

16. Ning G. Risk Evaluation of cAncers in Chinese diabeTic Individuals: a IONgitudinal (REACTION) study. J Diabetes. 2012;4(2):172-3.

17. Levey AS, et al. A new equation to estimate glomerular filtration rate. Ann Intern Med. 2009;150(9):604-12.

18. Stevens PE, Levin A. Evaluation and management of chronic kidney disease: synopsis of the kidney disease: improving global outcomes 2012 clinical practice guideline. Ann Intern Med. 2013;158(11):825-30.

19. Chang KT, et al. Which obesity index is the best predictor for high cardiovascular disease risk in middle-aged and elderly population? Arch Gerontol Geriatr. 2018;78:165-70.

20. Kouli GM, et al. Visceral adiposity index and 10-year cardiovascular disease incidence: The ATTICA study. Nutr Metab Cardiovasc Dis. 2017;27(10):881-9.

21. Randrianarisoa E, et al. Visceral Adiposity Index as an Independent Marker of Subclinical Atherosclerosis in Individuals Prone to Diabetes Mellitus. J Atheroscler Thromb. 2019;26(9):821-34.

22. Martens RJH, et al. Microvascular endothelial dysfunction is associated with albuminuria: the Maastricht Study. J Hypertens. 2018;36(5):1178-87.

23. Jones $\mathrm{CA}$, et al. Microalbuminuria in the US population: third National Health and Nutrition Examination Survey. Am J Kidney Dis. 2002;39(3):445-59.

24. Tanaka F, et al., Low-grade albuminuria and incidence of cardiovascular disease and all-cause mortality in nondiabetic and normotensive individuals. J Hypertens, 2016. 34(3): p. 506 - 12; discussion 512.

25. Jager $A$, et al. Microalbuminuria and peripheral arterial disease are independent predictors of cardiovascular and all-cause mortality, especially among hypertensive subjects: five-year follow-up of the Hoorn Study. Arterioscler Thromb Vasc Biol. 1999;19(3):617-24. 
26. Hillege $\mathrm{HL}$, et al. Urinary albumin excretion predicts cardiovascular and noncardiovascular mortality in general population. Circulation. 2002;106(14):1777-82.

27. Earle KA, et al. Microalbuminuria as a marker of silent myocardial ischaemia in IDDM patients. Diabetologia. 1996;39(7):854-6.

28. Yuyun MF, et al. Microalbuminuria and stroke in a British population: the European Prospective Investigation into Cancer in Norfolk (EPIC-Norfolk) population study. J Intern Med. 2004;255(2):24756.

29. Zander $\mathrm{E}$, et al. Peripheral arterial disease in diabetes mellitus type 1 and type 2: are there different risk factors? Vasa. 2002;31(4):249-54.

30. Hanai $\mathrm{K}$, et al. Involvement of visceral fat in the pathogenesis of albuminuria in patients with type 2 diabetes with early stage of nephropathy. Clin Exp Nephrol. 2010;14(2):132-6.

31. Kim JK, et al. Four-Year Changes in Visceral Fat Mass and the Risk of Developing Proteinuria in the General Population. PLoS One. 2015;10(6):e0131119.

32. Gambacciani $M$, et al. Body weight, body fat distribution, and hormonal replacement therapy in early postmenopausal women. J Clin Endocrinol Metab. 1997;82(2):414-7.

33. Ryan AS, Nicklas BJ, Berman DM. Hormone replacement therapy, insulin sensitivity, and abdominal obesity in postmenopausal women. Diabetes Care. 2002;25(1):127-33.

34. Camhi SM, et al. The relationship of waist circumference and BMI to visceral, subcutaneous, and total body fat: sex and race differences. Obesity (Silver Spring). 2011;19(2):402-8.

35. Mathieu P, et al. Visceral obesity and the heart. Int J Biochem Cell Biol. 2008;40(5):821-36.

36. Oh JY, Sung YA, Lee HJ. The visceral adiposity index as a predictor of insulin resistance in young women with polycystic ovary syndrome. Obesity (Silver Spring). 2013;21(8):1690-4.

\section{Figures}


Participants from 7 centers of Reaction study: Liaoning

10140, Gansu 10026, Guangzhou 9743, Sichuan 8105,

Shanghai 6821, Henan1978, Hubei 995

participants with the following diseases

were excluded

1. Kidney stones $(n=1541)$

2. Nephrotic syndrome $(n=17)$

3. Chronic nephritis $(\mathrm{n}=288)$

4. Kidney cyst $(n-573)$

5. Tumor $(n=1754)$

6. Fatty liver $(\mathrm{n}=4244)$

7. Viral hepatitis $(n=1539)$

8. Cirrhosis $(n=60)$

9. Diabetes $(n=7391)$

A total of 47808 participats aged over 40 years

were recruted

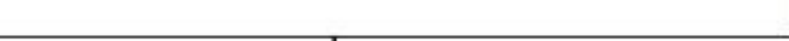

participants with the following previous

history of drugs used were excluded

1. ACEI drugs $(n=595)$

2. ARB drugs $\quad(n=1099)$

3. 1 ipid lowering drugs $(n=421)$

4. antihypertensive drugs $(n=7881)$

5. hypoglycemic drugs $(n=4799)$

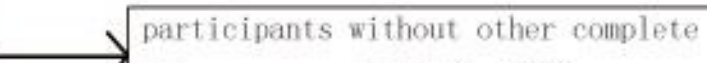

data were excluded $(n=1241)$

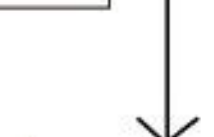

$\sqrt{ }$

28071 participants

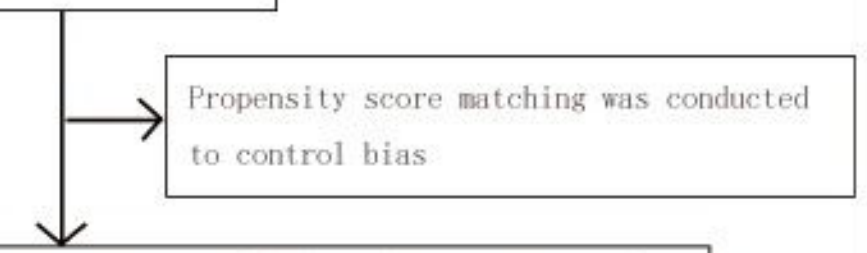

Finally, 24871 participants were included in the present study

\section{Figure 1}

Flow chart of the selection of the study participants. 


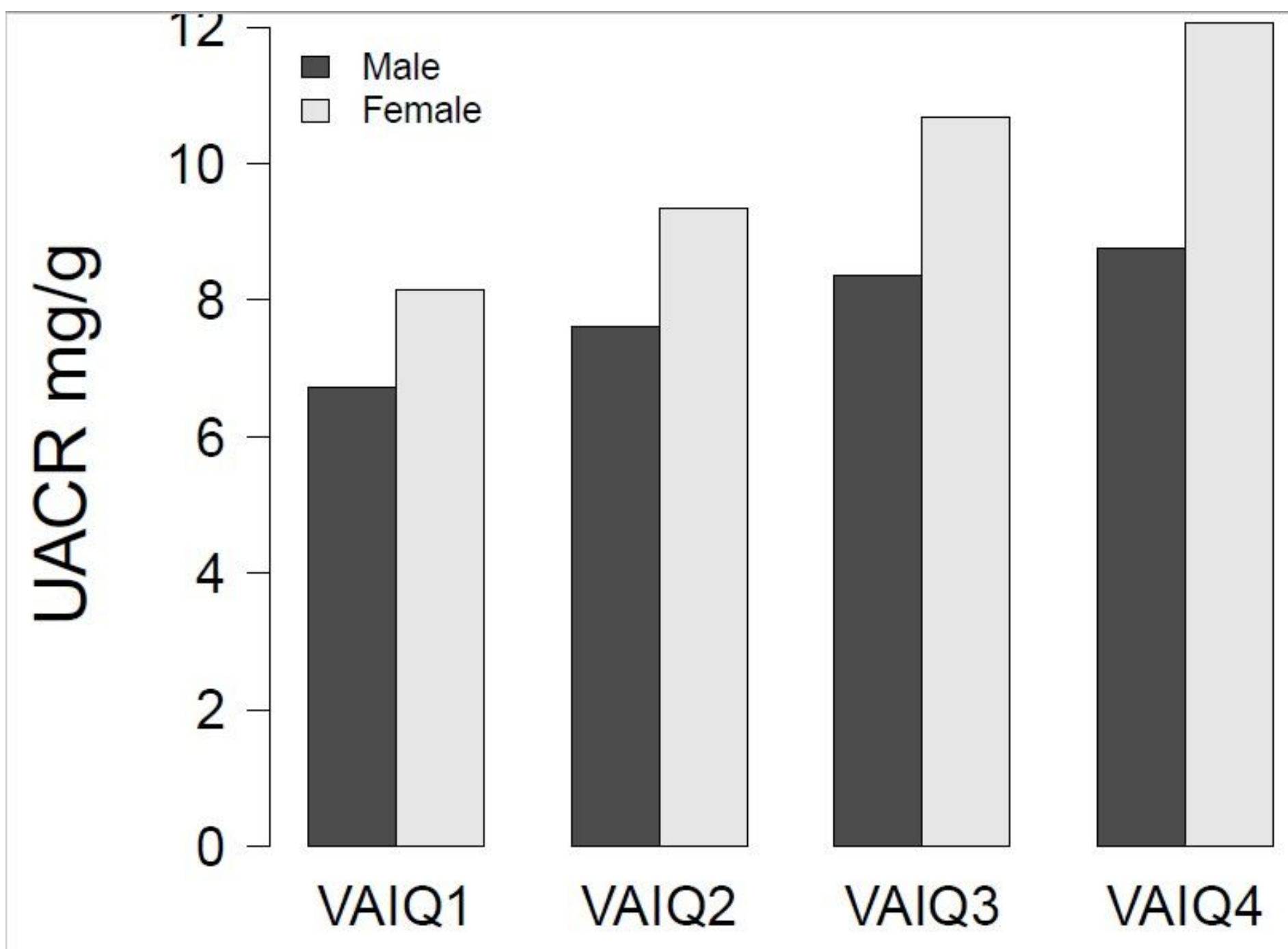

Figure 2

Associations of VAI with UACR by gender 